\title{
Improved limits on sterile neutrino mixing from a joint search of the MINOS, MINOS+, Daya Bay, and Bugey-3 experiments
}

\author{
Zhuojun $\mathrm{Hu}^{1, *}$ \\ Sun Yat-sen University, \\ No. 135, Xingang Xi Road, Guangzhou, PRC \\ E-mail: huzhj3@mail2.sysu.edu.cn
}

The Daya Bay, MINOS and MINOS+ experiments have searched for sterile neutrino mixing using electron antineutrino and muon (anti)neutrino disappearance, respectively, within a minimally extended four-neutrino scenario. They have recently combined their results, together with those from the Bugey-3 reactor neutrino experiment, to set the most stringent limits to date on the $\theta_{\mu e}$ mixing angle over five orders of magnitude in the sterile mass-squared difference $\left|\Delta m_{41}^{2}\right|$. The new constraints are significantly more stringent than the previous ones and exclude the sterileneutrino parameter space allowed by the LSND and MiniBooNE observations at 99\% C.L.(CL CL $_{s}$ for $\left|\Delta m_{41}^{2}\right|<1.2 \mathrm{eV}^{2}$, weakening the interpretation of these observations by the presence of a sterile neutrino. The result of the joint Daya Bay, MINOS, MINOS+ and Bugey-3 search along with a brief overview of the searches done by each experiment is presented.

40th International Conference on High Energy physics - ICHEP2020

July 28 - August 6, 2020

Prague, Czech Republic (virtual meeting)

${ }^{1}$ For the Daya Bay and MINOS/MINOS+ collaborations

* Speaker 


\section{Introduction}

Despite the success of the three-flavor neutrino mixing framework, some experimental observations, such as excesses of electron (anti-)neutrino events observed by LSND [1] and MiniBooNE [2], cannot be fully explained. One possible solution is mixing with a light sterile neutrino with masssquared difference $\Delta m_{41}^{2} \sim O(1) \mathrm{eV}^{2}$. The Daya Bay and MINOS experiments reported limits on light sterile neutrino mixing suggested by the LSND and MiniBooNE observations in 2016 [3]. An improved joint search utilizing electron antineutrino disappearance at Daya Bay and Bugey-3 and muon (anti)neutrino disappearance at MINOS/MINOS+ is described in this paper.

\section{Joint search for a light sterile neutrino}

The 3+1 model, with three active and one sterile neutrino, has been adopted in this analysis. In this model, the electron (anti)neutrino appearance probability at the baseline of LSND and MiniBooNE can be approximated by $4\left|U_{e 4}\right|^{2}\left|U_{\mu 4}\right|^{2} \sin ^{2}\left(\frac{\Delta m_{41}^{2} L}{4 E}\right)$, given $\left|\Delta m_{41}^{2}\right| \gg\left|\Delta m_{32}^{2}\right|$, where the appearance amplitude $4\left|U_{e 4}\right|^{2}\left|U_{\mu 4}\right|^{2}=\sin ^{2} 2 \theta_{14} \sin ^{2} \theta_{24} \equiv \sin ^{2} 2 \theta_{\mu e}$. On the other hand, the MINOS/MINOS+ and Daya Bay/Bugey-3 measurements constrain individual $\sin ^{2} \theta_{24}$ and $\sin ^{2} 2 \theta_{14}$, respectively. Hence through combination, strong constraints can be placed on the appearance amplitude $\sin ^{2} 2 \theta_{\mu e}$.
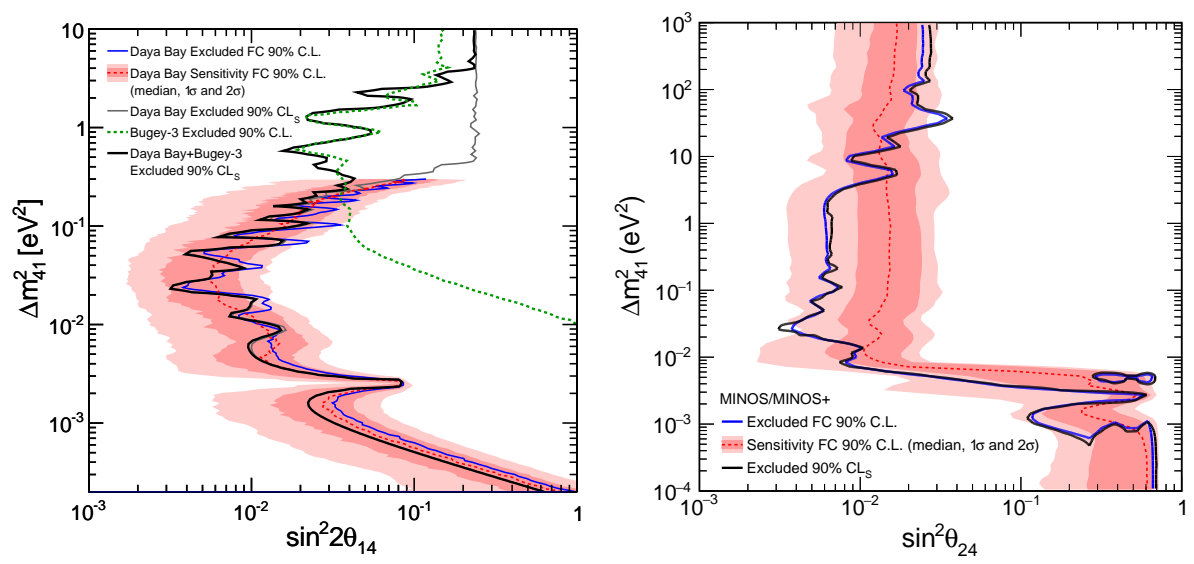

Figure 1: Left: The $\mathrm{CL}_{s}$ contours from Daya Bay data (grey) and its combination with the reproduced Bugey-3 (black), and the contour from Daya Bay data using the Feldman-Cousins method (blue). Right: The MINOS and MINOS+ exclusion contours [4] with the $\mathrm{CL}_{s}$ method (black) and the Feldman-Cousins method (blue). In both panels, the regions to the right of the curves are excluded at the $90 \%$ C.L. $\left(\mathrm{CL}_{s}\right)$. The $90 \%$ C.L. median sensitivity is shown in red along with the $1 \sigma$ and $2 \sigma$ bands.

Searches for light sterile neutrino mixing were performed at Daya Bay and MINOS/MINOS+ independently. The new search at Daya Bay advanced with $\sim 2$ times in exposure and reduction in some key systematic uncertainties. The MINOS/MINOS+ experiment used additional exposure of $5.80 \times 10^{20}$ POT and applied new analysis strategy: simultaneously fitting near and far spectra. Both experiments adopted two complementary methods, the Feldman-Cousins method and the $\mathrm{CL}_{s}$ method, to set exclusion limits on the light sterile neutrino mixing, as shown in Fig. 1. Daya Bay sets 
the world-leading limits on $\sin ^{2} 2 \theta_{14}$ for $2 \times 10^{-4} \mathrm{eV}^{2}<\left|\Delta m_{41}^{2}\right|<0.2 \mathrm{eV}^{2}$, while MINOS/MINOS+ places leading and stringent constraints on $\sin ^{2} \theta_{24}$ for values of $\Delta m_{41}^{2}>10^{-2} \mathrm{eV}^{2}$. Moreover, the Daya Bay and Bugey-3 combination allows one to set limits at higher $\Delta m_{41}^{2}$ region, and the resultant $\mathrm{CL}_{s}$ contour is presented in Fig. 1 on the left panel (black).

Following the same methodology as in the previous analysis [3], the disappearance measurements from MINOS, MINOS+, Daya Bay and Bugey-3 are combined to set limits on $\sin ^{2} \theta_{\mu e}$. The 99\% C.L. $\left(\mathrm{CL}_{s}\right)$ contour is shown in Fig. 2, in comparison with the appearance allowed regions.

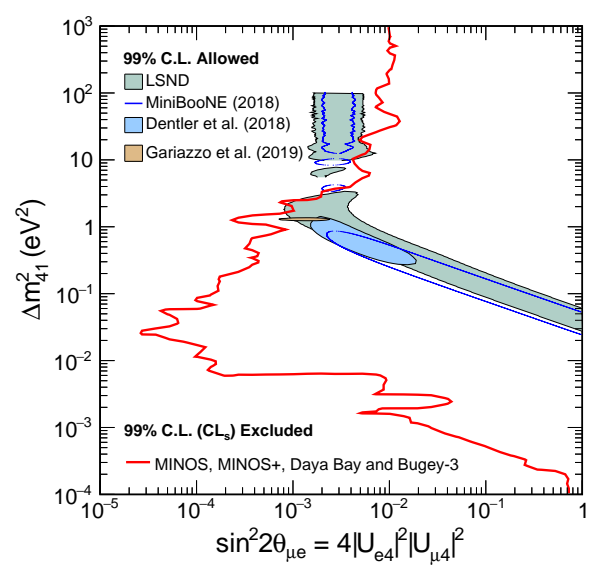

Figure 2: The combined limits at $99 \%$ C.L. $\left(\mathrm{CL}_{s}\right)$ on $\sin ^{2} 2 \theta_{\mu e}$ using the $\mathrm{CL}_{s}$ method (red). The exclusion contours are compared to the LSND and MiniBooNE allowed regions. Also shown are the allowed regions from global fits by Gariazzo et al. [5] and Dentler et al. [6].

\section{Conclusions}

Through combination, the most stringent constraints to date are set on $\sin ^{2} 2 \theta_{\mu e}$ over 5 orders of magnitude in the sterile neutrino mass-squared difference $\Delta m_{41}^{2}$. LSND and MiniBooNE allowed regions with $\Delta m_{41}^{2}<1.2 \mathrm{eV}^{2}$ are excluded at $99 \%$ C.L. $\left(\mathrm{CL}_{s}\right)$, including allowed regions from two fits to global data [5, 6]. Tension between the electron (anti)neutrino appearance indications and the null results from disappearance channels is increased.

\section{References}

[1] A. Aguilar-Arevalo et al. [LSND], Phys. Rev. D 64 (2001), 112007

[2] A. A. Aguilar-Arevalo et al. [MiniBooNE], Phys. Rev. Lett. 121 (2018) No.22, 221801

[3] P. Adamson et al. [Daya Bay and MINOS], Phys. Rev. Lett. 117 (2016) No.15, 151801

[4] P. Adamson et al. [MINOS+], Phys. Rev. Lett. 122 (2019) No.9, 091803

[5] S. Gariazzo, C. Giunti, M. Laveder and Y. F. Li, JHEP 06 (2017), 135

[6] M. Dentler, Á. Hernández-Cabezudo, J. Kopp, P. A. N. Machado, M. Maltoni, I. MartinezSoler and T. Schwetz, JHEP 08 (2018), 010 